\title{
Antimicrobial susceptibility pattern and multidrug resistance index in Pseudomonas aeruginosa among clinical isolates in Denizli, Turkey
}

\author{
SELMA KIRAC ${ }^{*}$, DILEK KESKIN ${ }^{2}$ and MURADIYE YARAR ${ }^{3}$ \\ ${ }^{1}$ Denizli Health Services Vocational High School, Pamukkale University, Denizli, Turkey \\ ${ }^{2}$ Cine Vocational High School, Adnan Menderes University, 09500-Cine-Aydin, Turkey \\ ${ }^{3}$ Denizli State Hospital, Denizli, Turkey
}

\begin{abstract}
Background: Pseudomonas aeruginosa is an important hospital infection agent causing morbidity and mortality with the ability to gain resistance to many antimicrobials. The objective of this study was to determine the sensitivity profiles of nosocomial $P$. aeruginosa isolates in Denizli, Turkey.

Methods: A total 120 P. aeruginosa strains which were isolated from specimens sent to the microbiology laboratory between January 2015 and December 2015 were investigated. Antimicrobial resistance was determined by agar disc diffusion method using Mueller-Hinton agar according to Clinical and Laboratory Standards Institute recommendations.

Results: With respect to sensitivity pattern, the most sensitive antimicrobials were Amikacin, colistin, tobramisin, netilmicin and gentamicin and the resistance rates were detected as $97 \%, 96 \%, 92 \%, 90 \%, 83 \%$, respectively over $120 \mathrm{P}$. aeruginosa strains. The sensitivity rates for the other antimicrobials were $56 \%$ for Piperacilin and 54\% for Tazobactam. P. aeruginosa strains $62(52 \%)$ isolates showed multiple antimicrobial resistance to 13 antimicrobials

Conclusion: To prevent the spread of the resistant bacteria, it is critically important to have strict antimicrobial policies while surveillance programmes for multidrug resistant organisms and infection control procedures need to be implemented. In the meantime, it is desirable that the antimicrobial susceptibility pattern of bacterial pathogens like $P$. aeruginosa in specialized clinical units to be continuously monitored and the results readily made available to clinicians so as to minimize the development of resistance.
\end{abstract}

Keywords: Pseudomonas aeruginosa, antimicrobial resistance, clinical isolates, Turkey

\section{Introduction}

Known for many years to be a cause of serious wound and surgical infections, but often regarded as a secondary or opportunistic invader rather than a cause of primary infection in healthy tissues, Pseudomonas aeruginosa has now clearly emerged as a major nosocomial pathogen in immunocompromised and debilitated patients, as well as in cystic fibrosis patients (Pier \& Ramphal, 2005). P. aeruginosa develops resistance to many antimicrobials and sometimes the sensitivity status can change during treatment. In particular, development of resistance is observed with the use of specific antimicrobial and resistant strains can be transmitted from patient to patient (Aloush et al., 2006). Multiple antimicrobial resistance (MAR) problems arise because of the combination of resistance against various antimicrobials used in therapy and cross resistance development between antimicrobials.

$P$. aeruginosa shows intrinsic and acquired resistance to many structurally unrelated antimicrobials, and previous exposure to antimicrobials often leads to multidrug-resistant $P$. aeruginosa strains (Mouton et al., 1993; Ciofu et al., 1994). When $P$. aeruginosa strains are resistant to antimicrobials, they increase the length of hospital stay and the cost of treatment (Kang et al., 2005). Increasing resistance through the use of false antibacterial agents presents serious problems in the treatment of infections (Mittal et al., 2009). Antimicrobial susceptibility data of $P$. aeruginosa is limited in Turkey (Berktaş et al., $2011 \mathrm{Er}$ et al., 2015). Because of these facts, it is of crucial importance to isolate and identify the offending strain in order for appropriate antimicrobial

\footnotetext{
*Correspondence E-mail: skirac@pau.edu.tr
} 
therapy to be initiated. The objective of this study was therefore, to determine the characteristics and patterns of antimicrobial resistance among isolates of $P$. aeruginosa recovered from clinical specimens in Denizli, Turkey.

\section{Materials and Methods}

\section{Culture and Identification}

This study was carried out in the Department of Microbiology, Denizli State Hospital, a centrally located medical centre in the Denizli city, between January 2015 and December 2015. Samples were collected from the hospitalized patients in different clinic parts of the hospital. Samples were taken from various sources like urine, broncho-alveolar lavage and tracheal aspirates.

The samples were streaked on nutrient agar plates and the plates were incubated at $37^{\circ} \mathrm{C}$ for 24 hours as described by Cheesborough (1985). Then the characteristic suspected single colonies were subjected to Gram's staining and then sub-cultured in MacConkey agar and blood agar. The pure isolates of Pseudomonas aeruginosa were transferred to $1 \%$ nutrient agar slant and stored in the refrigerator at $4^{\circ} \mathrm{C}$. P. aeruginosa was identified by biochemical test (sugar fermentation test) and biochemical tests were performed following the methods described in MacFadden (2000). Motility test of the isolated $P$. aeruginosa was performed following the method described by Cheesbrough (1985).

\section{Microbiological analyses and antimicrobial susceptibility testing}

P. aeruginosa was confirmed by the Vitek2 automated microbiology system (bioMérieux, Marcy l'Etoile, France). P. aeruginosa (ATCC 27853) was used as the quality control strain. The antimicrobials used were selected according to the 2004 National Committee for Clinical Laboratory Standard (NCCLS) guidelines: amikacin, colistin, tobramycin, netilmicin, gentamicin, aztreonam, cefepime, ceftazidime, levofloxacin, ciprofloxacin, imipenem, meropenem, tigecycline, piperacillin, piperacillin/tazobactam, tetracycline and sulfamethazol/trimetroprim.

\section{Antibiogram pattern of P. aeruginosa}

Antimicrobial resistance was determined by an agar disc diffusion test (Bauer et al., 1966) using Mueller-Hinton agar (Difco) according to Clinical and Laboratory Standards Institute (CLSI) recommendations (CLSI, 2005). Seventeen different antimicrobials were used. For antimicrobial resistance determination, the isolates were grown in Luria-Bertani (LB) broth until the turbidity equal to the $0.5 \mathrm{McFarland}$ standard (approximately $10^{8} \mathrm{cfu} / \mathrm{ml}$ ). Cultures were swabbed on to the

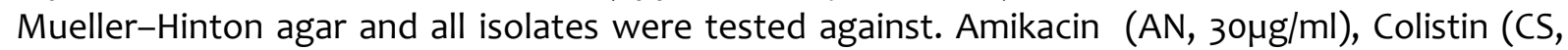

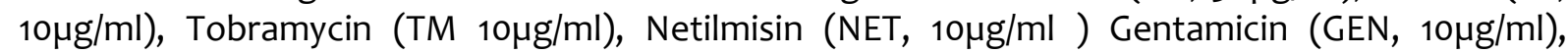

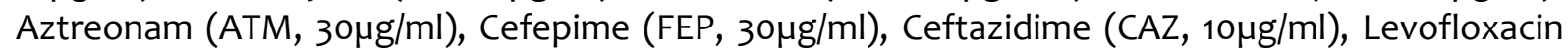

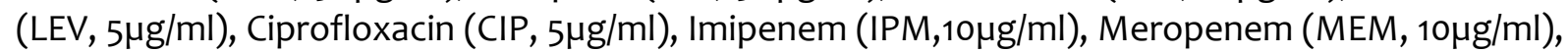

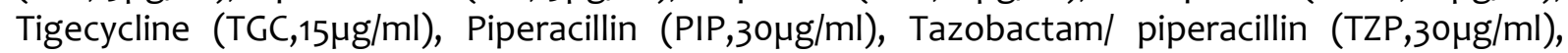
Tetracycline $(\mathrm{TE}, 30 \mu \mathrm{g} / \mathrm{ml})$, Sulfamethaxol/trimetroprim $(\mathrm{SXT}, 25 \mu \mathrm{g} / \mathrm{ml})$. The isolates grown in inoculation were evaluated as resistant and the others were evaluated as susceptible. The antibiotic discs were dispensed sufficiently separated from each other so as to avoid overlapping of inhibition zones. The plates were incubated at $37^{\circ} \mathrm{C}$, and the diameters of the inhibition zones were measured after $18 \mathrm{hr}$. All susceptibility tests were carried out in duplicate and were repeated twice if discordant results had been obtained.

\section{Multiple Antimicrobial Resistance Index}

For all isolates, we calculated the MAR index values (a/b, where "a" represents the number of antimicrobials the isolate was resistant to; and " $b$ " represents the total number of antimicrobials the isolate tested against). A MAR index value $\geq 0.2$ is observed when isolates are exposed to high risk sources of human or animal contamination, where antimicrobials use is common; in contrast a 
MAR index value $<$ or $=0.2$ observed when antimicrobials are seldom or never used (Krumperman, 1985; Matyar et al.,2008).

\section{Results}

A total of 120 isolates of P. aeruginosa were collected from January 2015 to December 2015 from different patients. With respect to sensitivity pattern, the most sensitive antimicrobials were Amikacin, colistin, tobramisin, netilmicin, gentamycin and the resistance rates were detected as $97 \%, 96 \%, 92 \%, 90 \%$, and $83 \%$, respectively. For the other antimicrobials, the sensitivity rates of $P$. aeruginosa were in the following order: Piperacilin and Tazobactam were recorded $56 \%$ and $54 \%$ respectively.

Table1. Antimicrobial susceptibility pattern of Pseudomonas isolated from clinical samples

\begin{tabular}{llll}
\hline Antimicrobial & Sensitivity & Resistance & Intermediate \\
\hline Amikacin & $116(97 \%)$ & $4(3 \%)$ & $0(0 \%)$ \\
Colistin & $115(96 \%)$ & $5(4 \%)$ & $0(0 \%)$ \\
Tobramycin & $110(92 \%)$ & $3(3 \%)$ & $7(5 \%)$ \\
Netilmicin & $108(90 \%)$ & $7(6 \%)$ & $5(4 \%)$ \\
Gentamicin & $99(83 \%)$ & $12(10 \%)$ & $9(8 \%)$ \\
Aztreonam & $87(73 \%)$ & $27(23 \%)$ & $6(5 \%)$ \\
Cefepime & $87(73 \%)$ & $11(9 \%)$ & $22(18 \%)$ \\
Ceftazidime & $85(71 \%)$ & $26(22 \%)$ & $9(8 \%)$ \\
Levofloxacin & $74(62 \%)$ & $44(37 \%)$ & $2(1 \%)$ \\
Ciprofloxacin & $73(61 \%)$ & $40(33 \%)$ & $7(6 \%)$ \\
Imipenem & $73(61 \%)$ & $47(39 \%)$ & $0(0 \%)$ \\
Meropenem & $69(57.5 \%)$ & $29(24.2 \%)$ & $26(22 \%)$ \\
Tigecycline & $70(58 \%)$ & $49(41 \%)$ & $1(1 \%)$ \\
Piperacillin & $67(56 \%)$ & $33(28 \%)$ & $20(17 \%)$ \\
Tozabactam/Piperacillin & $65(54.2 \%)$ & $29(24.2 \%)$ & $26(22 \%)$ \\
Tetracycline & $53(44.2 \%)$ & $67(56 \%)$ & $0(0 \%)$ \\
Sulfamethaxol/Trimethoprim & $8(7 \%)$ & $112(93 \%)$ & $0(0 \%)$ \\
\hline
\end{tabular}

Out of the 120 P. aeruginosa strains 62 (52\%) isolates showed Multiple Antibiotic Resistance (MAR) four to thirteen antimicrobials. The results were given Table2. The MAR indices ranged from 0.06 to 0.76 isl.

Table 2. Number of clinical samples and Multiple Antibiotic Resistance Index 120 Pseudomonas aeruginosa strains

\begin{tabular}{|c|c|c|}
\hline Clinical Samples & $\begin{array}{l}\text { No. of } \\
\text { isolates }\end{array}$ & Multiple Antimicrobial Resistance Index \\
\hline Urine & 38 & $\begin{array}{l}0.06 \text { (10 isl), } 0.12 \text { (5isl), } 0.18(4 i s l), 0.24(6 i s l), 0.29 \text { (4isl), } 0.35 \text { (2isl), } 0.41 \\
\text { (1isl), } 0.49(1 \mathrm{isl}), 0.53(1 \mathrm{isl}) 0.58(1 \mathrm{isl}), 0.65(1 \mathrm{isl}) 0.76(1 \mathrm{isl}), 0.70 \text { (1isl) }\end{array}$ \\
\hline Blood & 22 & $\begin{array}{l}0.06 \text { (5isl), } 0.12 \text { (2isl), } 0.18 \text { (2isl), } 0.24 \text { (3isl), } 0.29(3 i s l), 0.35(1 i s l), \\
0.41(2 i s l), 0.53 \text { (2isl), } 0.70 \text { (2isl) }\end{array}$ \\
\hline Abscess & 14 & $\begin{array}{l}0.06 \text { (1 isl), } 0.12 \text { (1isl), } 0.18 \text { (5isl), } 0.24 \text { (4isl), } 0.29 \text { (1isl), } 0.65 \text { (1isl) } \\
0.76(1 \mathrm{isl})\end{array}$ \\
\hline Tracheal aspirate & 37 & $\begin{array}{l}0.06 \text { (3isl), } 0.12 \text { (2isl), } 0.18 \text { (4isl), } 0.24 \text { (6isl), } 0.29 \text { (2isl), } 0.35 \text { (4isl), } 0.41 \\
\text { (1isl), } 0.47 \text { (5isl), } 0.59 \text { (4isl), } 0.65 \text { (3isl) } 0.70 \text { (3isl) }\end{array}$ \\
\hline Ear & 2 & 0.12 (1isl), 0.06 (1isl) \\
\hline Pleural fluid & 2 & 0.06 (1 isl), $0.18(1 \mathrm{isl})$ \\
\hline Cerebrospinal fluid & 1 & 0.65 (1isl) \\
\hline Mucus & 4 & 0.06 (1isl), 0.35 (1isl), 0.47 (1isl), 0.23 (1isl) \\
\hline
\end{tabular}

\section{Discussion}


Our results were similar to those of Jamasbi (2008) who reported sensitivity to Amikacin of $97 \%$. However other studies have reported lower rate of sensitivity (Sharma et al., 2010; Picao et al. 2008; Behera et al., 2008; Hocquet et al., 2007). An increased sensitivity of $P$. aeruginosa to Colistin was also seen in this study. Our results were similar to Kumar et al (2014). Tobramycin has a narrow spectrum of activity, but it is often used to eliminate $P$. aeruginosa in patients with cystic fibrosis (Hamed \& Deponnett, 2017). This aminoglycoside antimicrobial is commonly used to treat different Gram-negative bacteria (Bulitta et al., 2015) and has been reported to have good clinical outcome (Gonzalez \& Spencer, 1998). Sensitivity to Tobramycin was seen in $92 \%$ isolates in our study, while relatively lower rates of sensitivity have been observed in other studies elsewhere (Obritisch et al., 2004; Javiya et al. 2008; Franco et al., 2010). Sensitivity rate to aminoglycosides (gentamicin, netilmicin, amikac) in our study was high. Gentamicin has been used with excellent results in the treatment of sepsis due to Pseudomonas spp., in burn patients (Stone, 1966). Strateva et al. (2007) in Bulgaria reported higher resistance to P. Aeruginosa to aminoglycosides while Fadeyi et al., (2005) Nigeria reported relatively lower resistance levels.

Sensitivity rate of Aztreonam was showed in $73 \%$. Some researchers have reported Aztreonam sensitivity rate to $P$. aeruginosa in clinical samples (Gultekin et al., 2004; Durmaz- Çetin et al., 2004; Ersoz et al., 2004; Eksi et al., 2007; Kurtoglu et al., 2008; Gayyurhan et al., 2008; Tuncoglu et al., 2009). Cefepime is one of the few antimicrobials described to have constant antipseudomonal activity over the years, although publications on cefepime resistance are growing in number in recent years (Eksi et al., 2007; Gayyurhan et al., 2008; Pakoz et al., 2011; Ece et al.,2014; Kotwal et al.,2014). Our results were similar to Eksi et al (2007) who also reported sensitivity to Cefepime was $74.5 \%$.

Some researchers have reported ceftazidime sensitivity rate to $P$. aeruginosa in clinical samples (Yapar et al., 2000; Ayyıldiz et al., 2000; Demirci et al.,2001; Cesur et al.,2002; Al-Jasser \& Elkhizzi., 2004; Gultekin et al., 2004; Durmaz- Cetin et al., 2004; Ersoz et al., 2004; Ciftci et al., 2005; Yücel et al.,2006; Eksi et al., 2007; Gayyurhan et al.,2008; Kurtoglu et al., 2008; Afifi et al., 2013). Levofloxacin sensitivity rate to $P$. aeruginosa in clinical samples have been reported in a number of studies (Pakoz et al., 2011; Sen et al., 2014). Among the quinolones, Levofloxacin, a broad spectrum antimicrobial quinolones, is found to be effective against a variety of the clinical isolates, especially Enterococcus spp. and Pseudomonas aeruginosa (Fuchs et al., 1996; Hans et al., 1999).

Only $69(57.5 \%)$ isolates were susceptible to meropenem. Meropenem sensitivity rate to $P$. aeruginosa in clinical samples has been reported by other authors (Fidan et al., 2005; Gales et al., 2006; Gayyurhan et al.,2008; Somily et al., 2012; Afifi et al., 2013; Ece et al., 2014; Sen et al., 2014; Yayan et al., 2015). We found that $61 \%$ isolates were sensitive to Ciprofloxacin in our study, similar to other studies (Sharma et al., 2010; Javiya et al., 2008; Gokale \& Metgud, 2012). However, lower sensitivity rates have been reported by Franco et al. (2010) and Prakash \& Saxen (2013). Carbapenems are the drugs of choice for many infections caused by gram positive and gram negative bacteria (Nicolau 2008; Shah, 2008). Sensitivity to Imipenem was observed in $61 \%$ of isolates in our study. Much lower rates have been observed by others (Franco et al., 2010; Picao et al., 2008; Behera et al., 2008) while higher rate of sensitivity have been reported by Javiya et al. (2008) and by Hocquet et al. (2007).

Our rate of tigecycline sensitivity was $58 \%$. Similar sensitivity pattern has been reported elsewhere (Somily et al., 2012; Chaudhary et al., 2013). Sensitivity rate to P. aeruginosa to piperacillin found in our study are similar to others (Andrade et al., 2003; Gultekin et al., 2004; Al-Tawfig., 2007; Berktas et al., 2011; Yayan et al., 2015). The Piperacillin/Tazobactam combination was effective in only about half of the isolates which is comparable to that of Javiya et al. (2008), while higher sensitivity was reported by Hocquet et al. (2007). The sensitivity pattern of tetracycline and trimethoprim observed in our study has been reported by other researchers elsewhere (Eksi et al., 2007; Gayyurhan et al., 2008; Ullah et al.,2009; Rifaioglu et al., 2009; Sen et al.,2014; Toroglu et al.,2013; Sen et al.,2014 ). Tetracycline is a bacteriostatic antimicrobial and used to select mutants of multidrug resistance (Alonso et al., 1999). 
The MAR indices give an indirect suggestion of the probable source(s) of the organism. The MAR indices in this work were greater than 0.20, this confirms the report of Olayinka et al. (2004) that the MAR index greater than 0.20 indicates that the organisms must have been originated from an environment where antimicrobials are often used (Olayinka et al., 2004). Thus, the result of the MAR index in this work can be interpreted that these pathogens might have been originated from where these antimicrobials are used. The multidrug resistance of $P$. aeruginosa from the hospital was $11.10 \%$ which confirms the report of Hota et al. (2009) that outbreaks of multidrug-resistant $P$. aeruginosa colonization or infection can occur in urology wards, a burn unit, haematology/oncology units, and adult and neonatal critical care units and that various medical devices and environmental reservoirs can be implicated in the outbreaks of the pathogen.

To prevent the spread of the resistant bacteria, it is critically important to have strict antimicrobial policies while surveillance programmes for multidrug resistant organisms and infection control procedures need to be implemented. In the meantime, it is desirable that the antimicrobial susceptibility pattern of bacterial pathogens like $P$. aeruginosa in specialized clinical units to be continuously monitored and the results readily made available to clinicians so as to minimize the resistance.

\section{References}

Afifi, M.M, Suelam, I.I.A., Soliman, M.T.A. \& El-Gohary, M.G.S. (2013) Prevalence and antimicrobial susceptibility pattern of Pseudomonas aeruginosa isolated from environmental and clinical samples in Upper Egypt. International Journal of Biological Chemistry 7: 47-57.

Al-Jasser, A. \& Elkhizi, N.A. (2004) Antimicrobial susceptibility pattern of clinical isolates of Pseudomonas aeruginosa. Saudi Medical Journal 25: 780-784.

Alonso, A., Campanario, E. \& Martinez, J.S. (1999) Emergence of multidrug-resistant mutants is Increased under antimicrobial selective pressure in Pseudomonas aeruginosa. Microbiology 145: 2857-2862.

Aloush, V., Navon-Venezia, S., Seigman-lgra, Y., Cabili, S. \& Carmeli, Y. (2006) Multidrug-Resistant Pseudomonas aeruginosa: Risk Factors and Clinical Impact. Antimicrobial Agents \& Chemotherapy 50: 43-48.

Andrade, S.S., Jones, R.N., Gales, A.C. \& Sader, H.S. (2003) Increasing prevalence of antimicrobial resistance among Pseudomonas aeruginosa isolates in Latin American medical centres: 5 year report of the SENTRY Antimicrobial Surveillance Program (1997-2001). Journal of Antimicrobial Chemotherapy; London, 52: 140-1(2003).

Bauer, A.W., Kirby, W.M.M., Sherris, J.C. \& Turck, M. (1966) Antimicrobial susceptibility testing by a standardized single disc method. American Journal of Clinical Pathology 45: 493-496.

Behera, B., Mathur, P., Das, A., Kapil, A. \& Sharma, V. (2008) An evaluation of four different phenotypic techniques for detection of Metallo beta lactamase producing Pseudomonas aeruginosa. Indian Journal of Medical Microbiology 26:233-237.

Bulitta, J.B., Ly, N.S., Landersdorfer, C.B., Wanigaratne, N,A., Velkov, T., Yadav, R., Oliver, A., Martin, L., Shin, B.S., Forrest, A. \& Tsuji, B.T. (2015) Two mechanisms of killing of Pseudomonas aeruginosa by tobramycin assessed at multiple inocula via mechanism-based modeling. Antimicrobial Agents and Chemotherapy 59: 2315-2327(2015).

Cesur, S., Albayrak, F., Birengel, S., Kolcu, Z. \& Tekeli, E. (2002) Sensitivity of Pseudomonas aeruginosa isolated from different clinical samples to carpapenem and other beta lactam antimicrobials. Turkish Society of Microbiology 33:203.

Chaudhary, M., Kumar, S. \& Payasi, A. (2013) Prevalence and antimicrobial sensitivity of ExtendedSpectrum $\beta$-Lactamase producing gram negative bacteria from clinical settings in India from 2010-2012. International Journal of Medicine and Medical Sciences 46 (2).

Cheesbrough, M. (1985) Medical Laboratory Manual for Tropical Countries. Vol. II. 248-264 pp. 
Ciftci, I.H., Çetinkaya, Z., Aktepe, O.C., Arslan, F. \& Altındiş, M. (2005) Antimicrobial sensitivity of Pseudomonas aeruginosa isolated from clinical samples. Turkish Society of Microbiology 35:98-102.

Ciofu, O., Giwercman, B., Pedersen, S.S. \& Hoiby, N. (1994) Development of antimicrobial resistance in Pseudomonas aeruginosa during two decades of antipseudomonal treatment at the antipseudomonal treatment at the Danish CF Center. APMIS 102: 7-12.

CLSI (2005) Performance standards for antimicrobial susceptibility testing, 15th informational supplement. Document M 100-S 15. Clinical and Laboratory Standards Institute, Wayne, PA. Danish.

Demirci, M., Yorgancıgil, B. \& Arda, M. (2001) Sensitivity of Pseudomonas aeruginosa isolated from different clinical samples to some antimicrobials. Ankem Journal 15:30.

Durmaz-Çetin, B., Özcan, N., Oktar, M., Hamsan, H. \& Gül, M. (2004) The Change of Antimicrobial Resistance Rates of Pseudomonas aeruginosa Strains Isolated from Wounds and Abscesses in Three Years. Turkish Society of Microbiology 34:244-247.

Ece, G., Samlioglu, P., Atalay, S. \& Kose, S. (2014) Evaluation of the in vitro colistin susceptibility of Pseudomonas aeruginosa and Acinetobacter baumannii strains at a tertiary care centre in Western Turkey. Le Infezioni in Medicina 1:36-40.

Eksi, F., Bayram, A., Balcı, I. \& Özer, G. (2007) Investigation of induced beta-lactamse activity and antimicrobial resistance in Pseudomonas aeruginosa strains. Turkish Society of Microbiology 37: 142-146.

Fadeyi, A., Akanbi, A.A., Nwabuisi, C. \& Onile, B.A. (2005) Antimicrobial disc sensitivity pattern of Pseudomonas aeruginosa isolates obtained from clinical specimen in llorin, Nigeria. African Journal of Medicine and Medical Sciences 34: 303-306.

Fidan, I., Gürelik, F.Ç., Yüksel, S. \& Sultan, N. (2005) Antimicrobial Resistance of Pseudomonas aeruginosa Strains and Frequency of Metallo-Beta-Lactamases. Ankem Journal 19: 68-70.

Franco, M.R.G., Caiaffa-Filho, H.H., Burattini, M.N. \& Rossi, F. (20120) Metallo-beta-lactamases among imipenem-resistant Pseudomonas aeruginosa in a Brazilian university hospital. Clınıcs 65: 825-829.

Fuchs, P.C., Barry, A.L., Brown, S.D. (1996) AST surveillance group. Prevalence of resistance to three fluoroquinolones: Assessment of levofloxacin disk test error rates and surrogate predictors of levofloxacin susceptibility. Antimicrobial Agents and Chemotherapy 40:1633-1639.

Gales, A.C., Jones, R.N. \& Sader, H.S. (2006) Global assessment of the antimicrobial activity of polymyxin B against 54731 clinical isolates of Gram negative bacilli: report from the SENTRY antimicrobial surveillance programme (2001-2004). Clinical Microbiology \& Infection 12:315321.

Gayyurhan, E., Zer, Y., Mehli, M., Akgün, S. (2008) Determination of antimicrobial susceptibility and metallo- betalactamase production of Pseudomonas aeruginosa strains isolated in intensive care unit. Infection Journal 22: 49-52.

Gokale, S. \& Metgud, S.C. (2012) Chracterization and antimicrobial sensitivity pattern of nonfermenting gram negative bacilli from various clinical samples in a tertiary care hospital, Belgaum. Journal of Pharmaceutical and Biomedical Sciences 17:1-5.

Gonzalez, L.S. \& Spencer, J.P. (1998) Aminoglycosides: a practical review. Am. Fam. Physician., 58:1811-1820.

Hamed, K. \& Deponnett, L. (2017) Tobramycin inhalation powder: an efficient and efficacious therapy for the treatment of Pseudomonas aeruginosa infection in cystic fibrosis: a review based on clinical evidence. Therapeutic Advances in Respiratory Diseases 6: 121-137(2015).

Hans, H.S., Mary-Claude, N. \& Alain, J. (1999) Susceptibility to levofloxacin of clinical isolates of bacteria from intensive care and hematology/oncology patients in Switzerland: A multicentre study. Journal of Antimicrobial Chemotherapy 43: 51-54.

Hocquet, D., Berthelot, P., Roussel- Delvallez, M., Favre, R., Jeannot, K., Bajolet, O., Marty, N., Grattard, F., Mariani-Kurkdjian, P., Bingen, E., Husson, M., Couetdic, G. \& Plésiat, P. (2007) 
Pseudomonas aeruginosa may accumulate drug resistance mechanisms without losing its ability to cause blood stream infections. Antimicrobial Agents and Chemotherapy 353: 1-6.

Hota, S., Hirji, Z., Stockton, K., Lemieux, C., Dedier, H., Wolfaardt, G. \& Gardam, M.A.(2009) Outbreak of multidrug-resistant Pseudomonas aeruginosa colonization and infection secondary to imperfect intensive care unit room design. Journal of Infection Control and Hospital Epidemiology 30: 25-33.

Jamasbi, R.J. (2008) Phenotypic and genotypic characteristics of clinical isolates of Pseudomonas aeruginosa: rate of occurrence and distribution of different serotypes, antimicrobial susceptibility profiles, and molecular typing. Lab Medıcıne 39:155-161.

Javiya, V.A., Ghatak, S.B. \& Patel, J.A. (2008) Antimicrobial susceptibility patterns of Pseudomonas aeruginosa at a tertiary care hospital in Gujarat, India. Indian Journal of Pharmacology 40: 230-34.

Kang, C.I., Kim, S.H., Park, W.B., Lee, K.D., Kim, H.B., Kim, E.C., Oh, M.D. \& Choe, K.W. (2005) Risk factors for antimicrobial resistance and influence of resistance on mortality in patients with bloodstream infection caused by Pseudomonas aeruginosa. Microbial Drug Resistance 11: 68-74.

Kotwal, A., Biswas, D., Kakati, B., Thakuria, B. \& Bhardwaj, N. (2014) Efficacy of anti-pseudomonal antimicrobials: need to reconsider the empirical use of cefepime. Indian Journal of Medical Research 140: 560-562.

Krumperman, P.H. (1985) Multiple antibiotic resistance indexing of Escherichia coli to identify highrisk sources of fecal contamination of foods. Applied and Environmental Microbiology 46:165-170.

Kumar, R., Srivastava, P., Rishi, S., Dahiya, S., Hemwani, K. \& Nirwan, P.S. (2014) Detection and antimicrobial susceptibility pattern of Pseudomonas aeruginosa isolates in various clinical samples with special reference to metallo beta lactamase from a tertiary care hospital in Jaipur, India. National Journal of Medical Research 4: 128-131.

MacFadden, J.F. (2000) Biochemical tests for Identification of Medical Bacteria 3rd Ed. The Williams \& Wilkins Co., USA: 689-691pp.

Matyar, F., Kaya, A. \& Dinçer, S. (2008) Antibacterial agents and heavy metal resistance in Gramnegative bacteria isolated from seawater, shrimp and sediment in Iskenderun Bay, Turkey. Science of the Total Environment 407:279-285.

Mittal, R., Aggarwal, S., Sharma, S., Chhibber, S. \& Harjai, K. (2009) Urinary tract infections caused by Pseudomonas aeruginosa: A minireview. Journal of Infection and Public Health 2: 101-111.

Mouton, J.W., Den Hollander, J.G. \& Horrevorts, A.M. (1993) Emergence of antimicrobial resistance among Pseudomonas aeruginosa isolates from patients with cystic fibrosis. Journal of Antimicrobial Chemotherapy 31:919-926.

Nicolau, D.P. (2008) Carbapenems: a potent class of antimicrobials. Expert Opinion on Pharmacotherapy 9: 23-37.

Olayinka, A.T., Onile, B.A. \& Olayinka, B.O. (2004) Prevalence of multi-drug resistant (MDR) Pseudomonas aeruginosa isolates in serological units of Ahmadu Bello University teaching Hospital, Zaria Nigeria: An indication for effective control measures. Annals of African Medicine 3: 3-16.

Pakoz, N.I.E., Seriban-Dogan, S. \& Aral, M. (2011) Antimicrobial sensitivity of Pseudomonas aeruginosa isolated from different clinical samples to some antimicrobials. Ankem Journal 25:73-78.

Picão, R.C., Andarde, S.S., Nicoletti, A.G., Campana, E.H., Moraes, G.C., Mendes, R.E. \& Gales, A.C. (2008) Metallo beta lactamase detection: Compaarative evaluation of double disk synergy versus combined disk tests for IMP-, GIM-,SIM-,SPM-, or VIM- producing isolates. Journal of Clinical Microbiology 46: 2028-2037.

Pier, G. \& Ramphal, R. (2005) Pseudomonas aeruginosa. In: Mandell G, Bennett J, Dolin R, eds, Principles and practice of infectious diseases. Philadelphia, PA: Elsevier Churchill Livingstone; 2587-2615. 
Prakash, D. \& Saxen, R.S. (2013) Prevalence and antimicrobial susceptibility pattern of Pseudomonas aeruginosa isolated from urine samples of hospitalized patients in Meerut, Uttar Pradesh (India). World Journal of Pharmacy and Pharmaceutical Sciences 2: 275-286.

Sen, V., Aktar, F., Yolbaş, I., Tekin, R., Kelekçi, S., Tan, I., Bozkurt, F. \& Balik, H. (2014) Antimicrobial Susceptibility of Pseudomonas aeruginosa Strains in a University Hospital. Medical Research Journal 12:120-124.

Shah, P.M. (2008) Parenteral carbapenems. Clinical Microbiology and Infection 14:175-180.

Sharma, M., Yadav, S. \& Chaudhary, U. (2010) Metallo beta lactamase producing Pseudomonas aeruginosa in neonatal septicemia. Journal of Laboratory Physicians 2:14-16.

Somily, A.M., Absar, M.M. \& Arshad, M.Z. (2012) Antimicrobial susceptibility patterns of multidrugresistant Pseudomonas aeruginosa and Acinetobacter baumannii against carbapenems, colistin, and tigecycline. Saudi Medical Journal 33: 750-755.

Stone, H.H. (1966) Review of Pseudomonas sepsis in thermal burns: verdoglobin determination and gentamicin therapy. Annals of Surgery 163: 297-305.

Strateva, T., Ouzounova-Raykova, V., Markova B, Todorova, A., Marteva-Proevska, Y. \& Mitov, I. (2007) Problematic clinical isolates of Pseudomonas aeruginosa from the university hospitals in Sofia, Bulgaria: current status of antimicrobial resistance and prevailing resistance mechanisms. Journal of Medical Microbiology 56: 956- 963.

Toroglu, S., Avan, H. \& Keskin, D. (2013) Beta- Lactamases production and Antimicrobial resistance ratio of Pseudomonas aeruginosa from hospitalized patients in Kahramanmaraş, Turkey, Journal of Environmental Biology 34: 695-700.

Ullah, F., Malik, S.A. \& Ahmed, J. (2009) Antimicrobial susceptibility and ESBL prevalence in Pseudomonas aeruginosa isolated from burn patients in the North West of Pakistan. Burns 35: 1020-1025.

Yapar, N., Erdenizmenli, M., Gülay, Z, Küçükgüven-Biçmen, M., Yüce, A. \& Yulu, N. (2000) Antimicrobial resistance of Pseudomonas aeruginosa, Acinetobacter and Staphylococcus species. Infection Journal 14:507.

Yayan, J., Ghebremedhin, B. \& Rasche, K. (2015) Antimicrobial Resistance of Pseudomonas aeruginosa in Pneumonia at a Single University Hospital Center in Germany over a 10-Year Period. PLOS ONE 10(10): e0139836.

Yücel, M., Yavuz, T., Kaya, D., Behçet, M., Öztürk, C.E. \& Şahin, İ. (2006) The investigation of Changes antimicrobial resistance rates of Pseudomonas aeruginosa strains in years. Ankem Journal 20:152-155. 